\title{
Ovarian Stimulation with Urofollitropin (uFSH) Results in a Lower Yield of Oocytes Compared to Recombinant FSH (rFSH), Nevertheless, uFSH is at Least as Effective as rFSH in Younger Patients: Preliminary Results of a Retrospective Study with Antagonist Protocols in an IVF/ICSI Program
}

\author{
Peter Kemeter ${ }^{*}$, Monika Stroh-Weigert and Wilfried Feichtinger
}

Wunschbaby Institut Feichtinger, Vienna, Austria

\begin{abstract}
Objective: Differences in the mode of action between recombinant FSH (rFSH) and urinary derived FSH (uFSH) have been reported in cycles down-regulated by agonists. The aim of our study was to determine, if these differences also exist in cycles down-regulated by antagonists.
\end{abstract}

Method: Antagonist cycles performed between 2009 - 2012 were divided into two groups:

1. Cycles stimulated with rFSH preparations: Follitropin alfa $(n=203)$ and Follitropin beta $(n=443)$ and 2. Cycles stimulated with Urofollitropin $(\mathrm{n}=405)$.

Cetrorelix or Ganirelix were used as antagonists. All patients received 75 IU hMG additionally from day 6 of stimulation onwards up to the day of hCG administration.

A logistic regression analysis was conducted to find the most significant parameters predicting hCG-positive pregnancy for $2471 \mathrm{IVF}$ cycles. The results demonstrated that the predictors such as age of patients and number of cycles ever performed made negative contributions and number of oocytes retrieved and number of embryos transferred made positive contributions to prediction. Taking this into consideration, comparable groups could be created by including only firstever IVF cycles with more than 10 oocytes retrieved and 2 embryos transferred. Thus, the final sample for comparison included 98 patients in the rFSH-group and 27 patients in the uFSH group.

Results: There were no differences in basic personal data and gonadotropin consumption between the groups. Stimulation with rFSH resulted in a higher yield of oocytes compared to uFSH $(15,6$ vs. 14,4), however, the results of the following reproductive outcome parameters were all in favor of uFSH when $\mathrm{rFSH}$ and $\mathrm{uFSH}$ were compared: oocyte maturation rate ( $76.5 \%$ vs. $79.0 \%)$, fertilization rate $(53,6 \%$ vs. 58,6\%), embryo score 4 rate $(25,7 \%$ vs.. $31,1 \%)$, hCG-positive pregnancy rate $(43,9 \%$ vs. $59,3 \%)$, clinical pregnancy rate $(33,7 \%$ vs. $44,4 \%)$, embryo-cryopreservation rate $(19,3 \%$ vs. $30,5 \%)$ and abortion rate $(14,0 \%$ vs. $12,5 \%)$.

Conclusions: These results seem to support the concept that uFSH produces fewer oocytes than rFSH, but the oocytes produced by uFSH are, on an average, of better quality than those produced by rFSH. Basic studies have shown that different FSH isoforms with different elimination kinetics in the two gonadotropin preparations could be responsible for the different effects.

However, the results have to be confirmed by well designed prospective randomized studies.

Keywords: Urofollitropin, uFSH, rFSH, FSH glycosylation, IVF.

\section{BACKGROUND}

Ovarian stimulation with gonadotropin preparations has become an integral part of assisted reproduction treatment. At present, two recombinant follicle stimulating hormone $(\mathrm{rFSH})$ preparations are on the market: follitropin-alfa (Gonal-F, Serono) and follitropin-beta (Puregon, MSD). Both have been developed through genetic engineering, by trans-

*Address correspondence to this author at the Wunschbaby Institut Feichtinger, A-1130 Vienna, Lainzerstr. 6, Austria; Tel: + 436649224886;

Fax:+43125330334922; E-mail: peter.kemeter@wunschbaby.at fection of the human FSH gene into Chinese hamster ovary $(\mathrm{CHO})$ cells. The resulting clones are screened for FSH production and activity as well as genetic stability. A single cell is then selected and cultured to create a working cell bank (WCB) of identical cells that produce $\mathrm{rFSH}$ which is then harvested from the culture supernatant [1]. An alternative to $\mathrm{rFSH}$ is urinary FSH (uFSH), which is purified from the urine of post-menopausal women [2].

FSH is a complex glycoprotein composed of protein and carbohydrate. The protein part consists of two dissimilar non-covalently linked polypeptide subunits: alfa and beta [3], with the beta subunit conferring the biological specific- 
ity of FSH (the alfa subunit is common to all the pituitary glycoprotein hormones). The carbohydrate part consists of four side chains, referred to as glycans, each composed of varying numbers of sugars (oligosaccharides) and sialic acid. Due to their sialic acid content, more heavily glycosylated molecules have a more acidic isoelectric point (pI). Molecules with the same pI are referred to as isoforms, and several studies have shown that the anterior pituitary contains a spectrum of FSH isoforms with not only different isoelectric properties, but also different bioactivities and different circulating half-lives due to the micro-heterogeneity of the carbohydrate side-chains $[4,5]$. This mixture of different carbohydrate moieties produced by the pituitary gland is modulated by the ovulatory cycle: in the early follicular phase heavily sialylated, acidic isoforms with long half lives in vivo and low in-vitro biological potency predominate, whereas in the peri-ovulatory phase, the less-sialylated, less acidic isoforms, with short in vivo half-lives and high in-vitro biological activity are more prevalent. Based on these considerations, it has been assumed that the more acidic isoforms stimulate the follicular maturation process in a longer, but less intense manner, while the less acidic isoforms appearing to provide a short, but potent stimulus necessary for the induction of ovulation $[6,7]$.

Various studies have compared rFSH with Human Menopausal Gonadotropin (HMG) and more or less purified FSH preparations for ovarian stimulation in IVF/ICSI. While earlier studies reported $\mathrm{rFSH}$ to be more effective than HMG and/or uFSH [8-13], others found no significant difference [14-21], and some more recent studies have reported uFSH to be superior to rFSH [22-24] or "at least as effective" [25].

There is a highly-purified uFSH preparation (Fostimon; IBSA, Switzerland) that belongs to a new generation of urinary FSH preparations that have especially acidic glycosylation content. A number of advantages of this product over rFSH preparations have been reported, including: significantly more top-quality embryos [26]; consumption of significantly fewer units of FSH in patients older than 39, achieving equivalent embryo development and pregnancy rates [27]; significantly higher implantation and pregnancy rates when Fostimon was administered for the first 6 days, followed by $\mathrm{rFSH}$, compared to $\mathrm{rFSH}$ alone [28]; significantly less FSH consumption in patients achieving blastocyst transfers on day 5 [29]; and a significantly higher number of top quality embryos and transferring of cryopreserved embryos in PCO-patients [30].

Since these studies were all conducted using agonist cycles, and we predominantly use antagonist cycles, we were interested in determining whether the reported advantages of Urofollitropin could be achieved in antagonist cycles as well. Accordingly, this study reports a retrospective comparison of both rFSH preparations (Puregon ${ }^{\mathbb{B}}$, MSD and Gonal-F ${ }^{\mathbb{R}}$, Serono) with Fostimon ${ }^{B}$.

\section{DESIGN}

Patients were selected for the study if they had undergone IVF/ICSI treatments in our clinic between January 2009 and November 2012. Inclusion criteria were: spontaneous cycles; normal pap smear; normal vaginal and cervical bacteriology; negative tests for serum HIV, Hepatitis B and C, and syphi- lis; and hormone levels in the normal range $(\mathrm{FSH}<14$ $\mathrm{mIU} / \mathrm{ml}$, prolactin $<30 \mathrm{ng} / \mathrm{ml}, \mathrm{TSH}<2.0 \mathrm{uU} / \mathrm{ml}$ ). Exclusion criteria were abnormalities of the uterine cavity seen by sonography.

Patients were allocated to one of three groups (Figs. 1-3) by the physicians:

Group I = Follitropin-alfa $\left(\right.$ Gonal-F $\left.F^{\mathbb{R}}\right)+$ Cetrorelix $(\mathrm{Ce}-$
trotide $\left.^{\mathbb{R}}\right) ;$ Group $I I$
galutran Group III $=$ uFSH $\left(\right.$ Fostimon $\left.^{\circledR}\right)+$ Cetrorelix $\left(\right.$ Cetrotide $\left.^{\circledR}\right)$.

There was no randomization of patients, with doctors free to decide which treatment to prescribe. However, their decision may have been influenced by personal experience, price differences, product availability, information from the pharmaceutical companies, seminars, etc.

To prime menstruation, all patients received tablets prepared by the pharmacist containing $2 \mathrm{mg}$ norethisterone acetate and $0.01 \mathrm{mg}$ ethinyl estradiol, three times daily for 10 days, starting on a Friday between day 16 and 22 of the cycle, with the final dose on a Sunday. At the same time, patients received prednisolone $(2.5 \mathrm{mg}$ in the morning and 5 $\mathrm{mg}$ in the evening). This adjuvant mild corticoid therapy (which was continued throughout the treatment cycle and up to the ninth week of pregnancy), has been found to suppress excessive adrenal androgen production, which can affect ovarian steroid metabolism [31, 32]. In addition to the different FSH preparations, all the patients received an injection containing 75 IU FSH and 75 IU LH (Merional ${ }^{\circledR}$ ) daily from stimulation day 6 until the day of hCG administration, to avoid a possible lack of LH caused by the antagonist. Exogenous LH supplementation has been shown to benefit patients older than 35 years of age [33-36], and patients with a sub-optimal response to FSH-only preparations (poor responders) [37-40].

In all groups, ovarian stimulation commenced on the Friday after the last dose of $2 \mathrm{mg}$ norethisterone acetate and $0.01 \mathrm{mg}$ ethinyl estradiol (Figs. 1-3). In Group I, daily doses of Gonal- $F^{\circledR}$ were 225 IU up to day 5 , then 150 IU from day 6 to the day of hCG administration. Cetrotide ${ }^{(\mathbb{B}}(0.25$ $\mathrm{mg} /$ day) and Merional ${ }^{\mathbb{B}}$ were administered from day 6 (Fig. 1). The other groups followed a similar protocol, with Puregon ${ }^{\mathbb{B}}$ instead of Gonal- $F^{\circledR}$, and Orgalutran ${ }^{\circledR}$ instead of Cetrotide $^{\circledR}$ for Group II (Fig. 2), and Fostimon ${ }^{\circledR}$ and Cetrotide ${ }^{\circledR}$ for Group III (Fig. 3). During the luteal phase, patients received micronized progesterone $\left(\right.$ Utrogestan $^{\mathbb{B}}$, Meda Pharma, Austria; taken orally: $3 \times 200 \mathrm{mg} /$ day), together with $20 \mathrm{mg}$ dydrogesterone (Duphaston ${ }_{(\mathbb{B}}$ Solvay, Austria) and $2 \mathrm{mg}$ estradiol valerate (Progynova ${ }^{\circledR}$, Schering, Germany) up to week 9 of pregnancy.

All patients signed an informed consent that they agreed to the inclusion of their data in the analysis.

The study was approved by the Ethics Committee of the City of Vienna, Austria (reference: EK_12_177_1012_Votum_positive, approved 09 November 2012). 


\section{Stimulation-protocol Group I: Gonal-F + Cetrotide}

NORETHISTERONE ACETATE $0,6+$ EE 0,03 on days $18-27$ the cycle before

\begin{tabular}{|l|c|c|c|c|c|c|c|c|c|c|c|}
\hline Treatment Day & 1 & 2 & 3 & 4 & 5 & 6 & 7 & 8 & 9 & 10 & 11 \\
\hline Day of the week & FR & SA & SU & MO & TUE & WED & THU & FR & SA & SU & MO \\
\hline Date & & & & & & & & & & & \\
\hline Sonography & & & & & & & & So & & & \\
\hline Prednisolone 5 mg & \multicolumn{7}{|c|}{$1 / 2$ tablet in the morning, 1 tablet in the evening } \\
\hline GONAL-f 75 I.U. & 3 & 3 & 3 & 3 & 3 & 2 & 2 & & & & \\
\hline MERIONAL 75 I.U. & 0 & 0 & 0 & 0 & 0 & 1 & 1 & & & & \\
\hline CETROTIDE 0,25 mg & & & & & & 1 & 1 & & & & \\
\hline $\begin{array}{l}\text { Partner: } \\
\text { Zithromax 500 mg }\end{array}$ & 1 & 1 & 1 & & & & & & & & \\
\hline
\end{tabular}

Kemeter P. et al. - Wunschbaby-Zentrum Vienna

Fig. (1). Antagonist protocol with Gonal-f and Cetrotide, allocating the dosages of the preparations to the stimulation days.

\section{Stimulation-protocol Group II: Puregon + Orgalutran}

NORETHISTERONE ACETATE $0,6+$ EE 0,03 on days $18-27$ the cycle before

\begin{tabular}{|l|c|c|c|c|c|c|c|c|c|c|c|}
\hline Treatment Day & 1 & 2 & 3 & 4 & 5 & 6 & 7 & 8 & 9 & 10 & 11 \\
\hline Day of the week & FR & SA & SU & MO & TUE & WED & THU & FR & SA & SU & MO \\
\hline Date & & & & & & & & & & & \\
\hline Sonography & & & & & & & & So & & & \\
\hline Prednisolone 5 mg & \multicolumn{7}{|c|}{$1 / 2$ tablet in the morning, 1 tablet in the evening } \\
\hline PUREGON 75 I.U. & 3 & 3 & 3 & 3 & 3 & 2 & 2 & & & & \\
\hline MERIONAL 75 I.U. & 0 & 0 & 0 & 0 & 0 & 1 & 1 & & & & \\
\hline ORGALURAN 0,25 mg & & & & & & 1 & 1 & & & & \\
\hline $\begin{array}{l}\text { Partner: } \\
\text { Zithromax 500 mg }\end{array}$ & 1 & 1 & 1 & & & & & & & & \\
\hline
\end{tabular}

Kemeter P. et al. - Wunschbaby-Zentrum Vienna

Fig. (2). Antagonist protocol with Puregon and Orgalutran, allocating the dosages of the preparations to the stimulation days. 


\section{Stimulation-protocol Group III: Fostimon + Cetrotide}

NORETHISTERONE ACETATE 0,6 + EE 0,03 on days $18-27$ the cycle before

\begin{tabular}{|l|c|c|c|c|c|c|c|c|c|c|c|}
\hline Treatment Day & 1 & 2 & 3 & 4 & 5 & 6 & 7 & 8 & 9 & 10 & 11 \\
\hline Day of the week & FR & SA & SU & MO & TUE & WED & THU & FR & SA & SU & MO \\
\hline Date & & & & & & & & & & & \\
\hline Sonography & & & & & & & & So & & & \\
\hline Prednisolone 5 mg & \multicolumn{7}{|c|}{$1 / 2$ tablet in the morning, 1 tablet in the evening } \\
\hline FOSTIMON 75 I.U. & 3 & 3 & 3 & 3 & 3 & 2 & 2 & & & & \\
\hline MERIONAL 75 I.U. & 0 & 0 & 0 & 0 & 0 & 1 & 1 & & & & \\
\hline CETROTIDE 0,25 mg & & & & & & 1 & 1 & & & & \\
\hline $\begin{array}{l}\text { Partner: } \\
\text { Zithromax 500 mg }\end{array}$ & 1 & 1 & 1 & & & & & & & & \\
\hline
\end{tabular}

Kemeter P. et al. - Wunschbaby-Zentrum Vienna

Fig. (3). Antagonist protocol with Fostimon and Cetrotide, allocating the dosages of the preparations to the stimulation days.

\section{ANALYSIS}

The primary end point was the hCG-positive pregnancy rate (hCG level $>10 \mathrm{mU} / \mathrm{ml} 15$ days after oocyte retrieval). Secondary end points were: number of oocytes retrieved; number of mature oocytes (MII); fertilization rate; embryo quality score 4 [41]; sonographic endometrial thickness on the day of hCG administration; clinical pregnancy rate (defined as fetal heart activity at 6-8 weeks gestation); and implantation rate (fetal heart activity/transferred embryo).

A standard statistical program was used for data analysis (SPSS/PC +4.0). Groups were compared using the $\chi^{2}$-test for binary data, as well as the two-tailed Students $t$ tests for the continuous data. $\mathrm{P}$ values of $<0.05$ were considered to indicate a statistically significant difference.

A logistic regression analysis was conducted to predict hCG-positive pregnancy for $2471 \mathrm{IVF}$ cycles using the age of the patients (age), the number of retrieved oocytes (oocytes) and replaced embryos (embryos transferred) as well as the total number of IVF cycles (cycles-ever) and the number of IVF cycles performed just in our clinic (WIF cycles) as predictors. A test of the full model against a constant only model was statistically significant, indicating that the predictors as a set reliably distinguished between pregnant and not pregnant patients (chi square $=383.991, \mathrm{p}$ $<.000$ with $\mathrm{df}=5$ ).

The Wald criterion demonstrated that the predictors age and cycles- ever made a significant negative contribution ( $p$ $<0.001, \mathrm{p}=0.003$, resp.), while embryos transferred made a significant positive contribution $(\mathrm{p}<0.001)$ to prediction, and oocytes correlated positively on a trend level $(\mathrm{p}=0,076)$ while WIF cycles correlated insignificantly positively with hCG-positive pregnancy $(\mathrm{p}=0.19$, Table $\mathbf{1})$.

Group I and Group II were aggregated to the rFSH-group which was compared with Group III, the uFSH-group (Table 3).

In order to find comparable $\mathrm{rFSH}$ and uFSH groups, we avoided the confounding effects of cycles- ever and embryos transferred by including only first-ever IVF patients into the study in whom two embryos had been transferred. This process reduced the number of cases to 211 in the $\mathrm{rFSH}$ group and 81 in the uFSH group (Tables $\mathbf{2}$ and $\mathbf{3}$ ).

In addition, age was stratified into the following groups by: (1) up to 25 years; (2) 26-30 years; (3) 31-35 years; (4) 36-40 years; and (5) $41+$ (41 years or older) (Fig. 4). Within these strata the age differences between the $\mathrm{rFSH}-$ and uFSH-group were minimal and could be neglected (Fig 4a).

Finally, only those patients were included into the study in which at least 11 oocytes had been retrieved. By this way the final sample included 98 cases in the rFSH-group and 27 cases in the $\mathrm{uFSH}$-group. These groups were comparable in regard to the four confounding factors listed in Table $\mathbf{1}$.

\section{RESULTS}

Table 2 shows the results of the three groups. It is obvious that Group III was significantly different from the other groups: patients in Group III were older, produced fewer oocytes and got fewer embryos replaced. Given these large differences, the differences in reproductive outcome seemed 
Table 1. Logistic Regression Analysis using 5 predictors of hCG-positive pregnancy

\begin{tabular}{|c|c|c|c|c|c|c|c|}
\hline \multicolumn{7}{|c|}{ Nogistic Regression Analysis } \\
\hline \multicolumn{7}{|c|}{ Dependent Variable: hCG-positive pregnancy } \\
\hline \multicolumn{7}{|c|}{ Variables in the Equation } \\
\hline \multicolumn{7}{|c|}{ Sumber of cases included: 2471 } \\
\hline Variables & B & S.E. & Wald & df & Sig & R & Ecp(B) \\
\hline \hline Age &,- 0860 &, 0094 & 84,1019 & 1 &, 0000 &,- 1633 &, 9176 \\
\hline Embryos transferred &, 8385 &, 0675 & 154,1483 & 1 &, 0000 &, 2224 & 2,3130 \\
\hline Cycles ever &,- 1645 &, 0458 & 12,8894 & 1 &, 0003 &,- 0595 &, 8483 \\
\hline Oocytes &, 0160 &, 0090 & 3,1494 & 1 &, 0760 &, 0193 & 1,0162 \\
\hline Cycles at WIF &, 0726 &, 0560 & 1,6812 & 1 &, 1948 &, 0000 & 1,0753 \\
\hline Constant &, 9679 &, 3514 & 7,5893 & 1 &, 0059 & \\
\hline
\end{tabular}

Table 2. Results of Antagonist Protocols for First-Ever IVF Cycles

\begin{tabular}{|c|c|c|c|c|}
\hline & Group I & Group II & Group III & $\mathbf{P}$ \\
\hline Patients (n) & 105 & 243 & 175 & \\
\hline Age $(\mathrm{X} \pm \mathrm{SD})$ & $33.3 \pm 5.0$ & $34.6 \pm 4.9$ & $38.0 \pm 5.5$ & $\begin{array}{c}\mathbf{0 , 0 2 4} \text { (I vs II) } \\
<\mathbf{0 , 0 0 1}(\text { I,II vs III) }\end{array}$ \\
\hline Oocytes $(\mathrm{X} \pm \mathrm{SD})$ & $10.4 \pm 5.6$ & $9.9 \pm 6.2$ & $7.1 \pm 5.0$ & $<\mathbf{0 , 0 0 1}$ (I, II vs III) \\
\hline Mature (MII) Oocytes (\%) & $78.1 \pm 18.5$ & $76.0 \pm 18.8$ & $78.8 \pm 23.0$ & NS \\
\hline Fertil. 2 PN (\%) & $56.6 \pm 21.8$ & $53.6 \pm 22.4$ & $55.7 \pm 28.9$ & NS \\
\hline Fertil. 2 PN ICSI (\%) & $72.1 \pm 22.8$ & $69.4 \pm 25.1$ & $69.0 \pm 29.8$ & NS \\
\hline Embryo Score $4(\%)$ & $30.6 \pm 28.1$ & $26.7 \pm 29.6$ & $28.6 \pm 33.1$ & NS \\
\hline Transferred embryos $(\mathrm{X} \pm \mathrm{SD})$ & $1.7 \pm 0.7$ & $1.7 \pm 0.8$ & $1.4 \pm 0.9$ & $\begin{array}{l}\mathbf{0 . 0 0 3} \text { (I vs III) } \\
\mathbf{0 . 0 0 2} \text { (II vs III) }\end{array}$ \\
\hline Endometrial thickness $(\mathrm{X} \pm \mathrm{SD})$ & $11.0 \pm 2.0$ & $10.8 \pm 1.9$ & $10.7 \pm 2.1$ & NS \\
\hline Pregnancy rate $(\%)$ & $40.0 \pm 49.2$ & $34.6 \pm 47.7$ & $30.3 \pm 46.1$ & NS \\
\hline Implantation rate (\%) & $25.1 \pm 39.3$ & $18.6 \pm 33.7$ & $16.8 \pm 32.8$ & NS \\
\hline Clinical pregnancy rate $(\%)$ & $33.3 \pm 47.4$ & $25.9 \pm 43.9$ & $20.6 \pm 40.5$ & $\mathbf{0 . 0 1 7}$ (I vs III) \\
\hline Abortion rate (\%) & $16.7 \pm 37.7$ & $19.0 \pm 39.5$ & $15.1 \pm 36.1$ & NS \\
\hline Cryopreserved embryos (\%) & $19.1 \pm 44.0$ & $15.9 \pm 24.8$ & $10.9 \pm 23.9$ & NS \\
\hline
\end{tabular}

to be surprisingly low. The mean levels of the pregnancy and implantation rate were insignificantly lower than in the other groups and only the clinical pregnancy rate was significantly lower compared to Group I, but the difference to Group II was not significant. Groups I and II were - except for the small but significant age difference - comparable (Table 1).

In Table 3 the results of first-ever IVF cycles with 2 embryos transferred are presented.

Patients in the uFSH-group were still significantly older and produced significantly fewer oocytes than those in the rFSH-group ( $\mathrm{p}=0,005$ and 0,009 , respectively). The other parameter, however, showed fairly comparable mean levels without significant differences.

In addition, Fig. (4a) illustrates the age distribution of the sample of Table $\mathbf{3}$ across the five age-groups. Fig. $\mathbf{4 b}$ evidently shows that there were fewer oocytes in the uFSHgroup compared to the rFSH group and the difference was based on the whole sample - highly significant $(p=0.009$, Fig. 4b). However, considering only the younger patients up to 35 years, the difference did not reach significance $(p=0.272)$. On the other hand, higher rates of mature and fertilized oocytes and of pregnancies and implantations were 
Table 3. Results of Antagonist Protocols for First-Ever IVF Cycles with 2 Embryos Transferred, Stimulated with either rFSH or uFSH

\begin{tabular}{|c|c|c|c|}
\hline & rFSH Group & uFSH Group & $\mathbf{P}$ \\
\hline Patients (n) & 211 & 81 & \\
\hline Age $(\mathrm{X} \pm \mathrm{SD})$ & $34.1 \pm 4.6$ & $35.9 \pm 4.9$ & 0.005 \\
\hline Oocytes $(\mathrm{X} \pm \mathrm{SD})$ & $10.8 \pm 5.4$ & $9.0 \pm 4.5$ & 0.009 \\
\hline Mature (MII) Oocytes (\%) & $78.9 \pm 15.9$ & $81.1 \pm 18.2$ & NS \\
\hline Fertil. 2 PN (\%) & $58.0 \pm 17.9$ & $61.0 \pm 20.0$ & NS \\
\hline Fertil. 2 PN ICSI (\%) & $73.4 \pm 17.5$ & $76.3 \pm 18.1$ & NS \\
\hline Embryo Score $4(\%)$ & $30.1 \pm 28.3$ & $30.8 \pm 30.0$ & NS \\
\hline Endometrial thickness $(\mathrm{X} \pm \mathrm{SD})$ & $10.7 \pm 2.0$ & $10.8 \pm 2.0$ & NS \\
\hline Pregnancy rate $(\%)$ & $44.1 \pm 49.8$ & $48.1 \pm 50.3$ & NS \\
\hline Implantation rate (\%) & $19.7 \pm 30.5$ & $19.1 \pm 32.2$ & NS \\
\hline Clinical pregnancy rate (\%) & $32.7 \pm 47.0$ & $32.1 \pm 47.0$ & NS \\
\hline Abortion rate (\%) & $19.4 \pm 39.7$ & $17.9 \pm 38.9$ & NS \\
\hline
\end{tabular}

observed much more often in the uFSH-group compared to the rFSH group in these younger patients (Figs. 4c-g).

The final comparison of rFSH with uFSH of first-ever cycles with more than 10 oocytes retrieved and two embryos replaced didn't show significant differences between the groups in terms of BMI, cause of infertility, duration of infertility, cigarette smoking, or consumption of FSH and HMG (Table 4). And there were no significant differences in the results either, however, it was remarkable, that even though the uFSH-group had a somewhat lower number of oocytes compared to the rFSH-group, the rate of mature oocytes, the fertilization rate, the rate of embryos score 4 , the hCG-positive pregnancy rate, the clinical pregnancy rate and the rate of cryopreserved embryos were all higher in the uFSH-group, and the abortion rate was lower compared to the rFSH-group (Table 5).

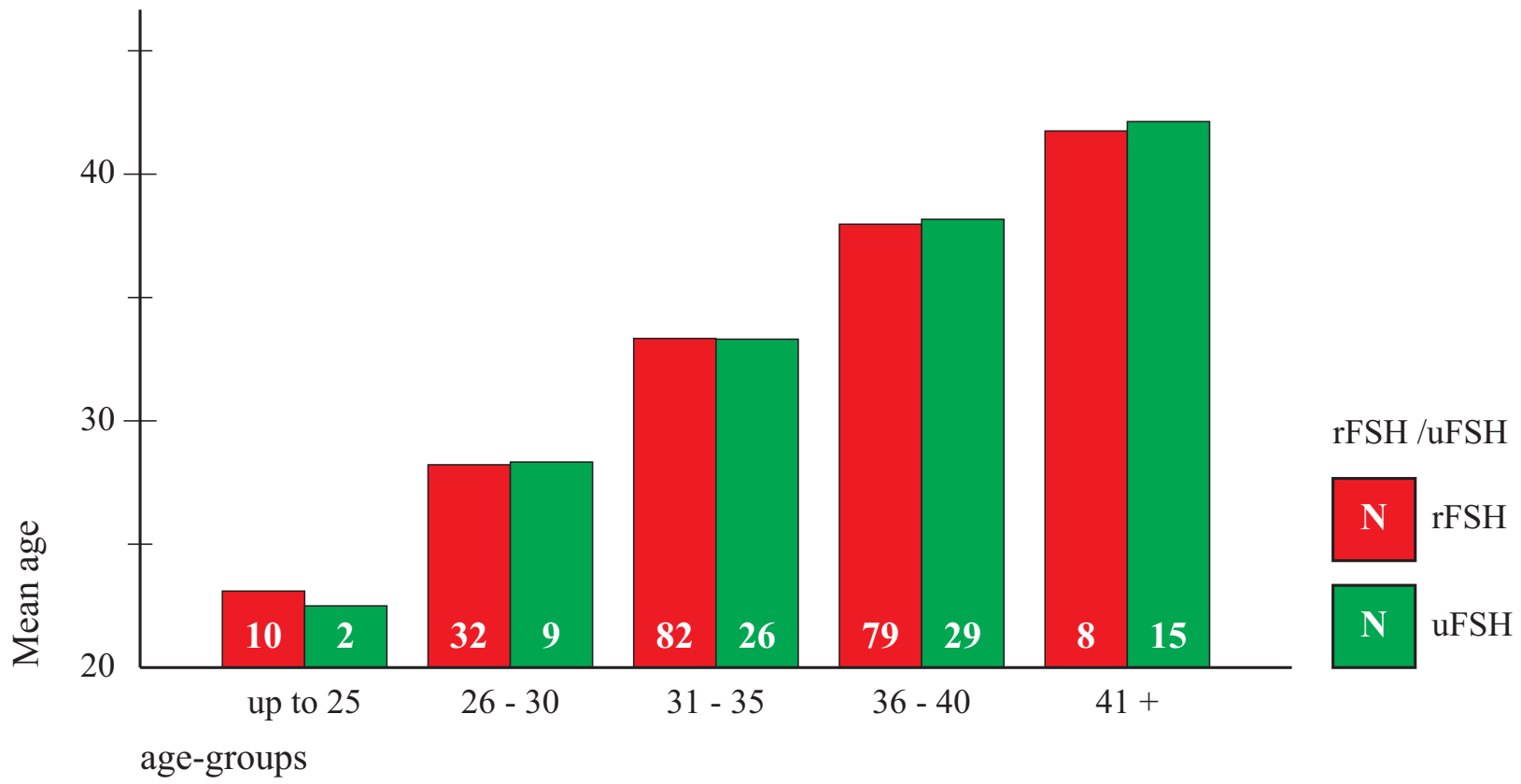

Fig. (4a). Comparison of $\mathrm{rFSH}$ and $\mathrm{uFSH}$ groups in regard to the age of patients.

Within the age strata there were only minimal differences in age between the treatment Groups 
Fig. (4). Contd....

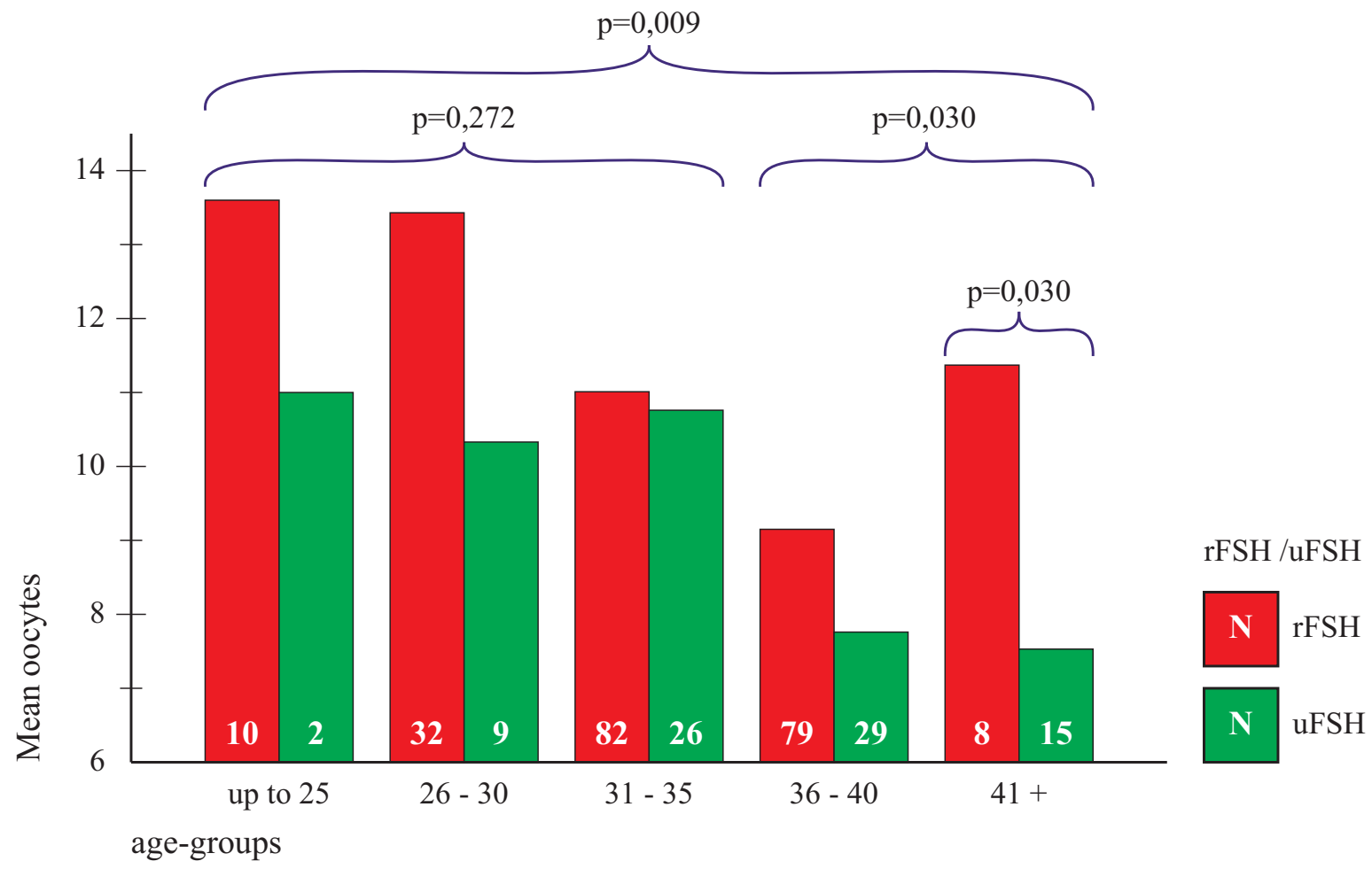

Fig. (4b). Comparison of the number of oocytes retrieved after rFSH or uFSH stimulation.

In the group as a whole, significantly fewer oocytes were retrieved in the $\mathrm{uFSH}$ group compared to the $\mathrm{rFSH}$ group ( $\mathrm{p}=0,009$ ), however, the difference was not significant in younger patients between 25 and 35 years $(p=0,272)$.

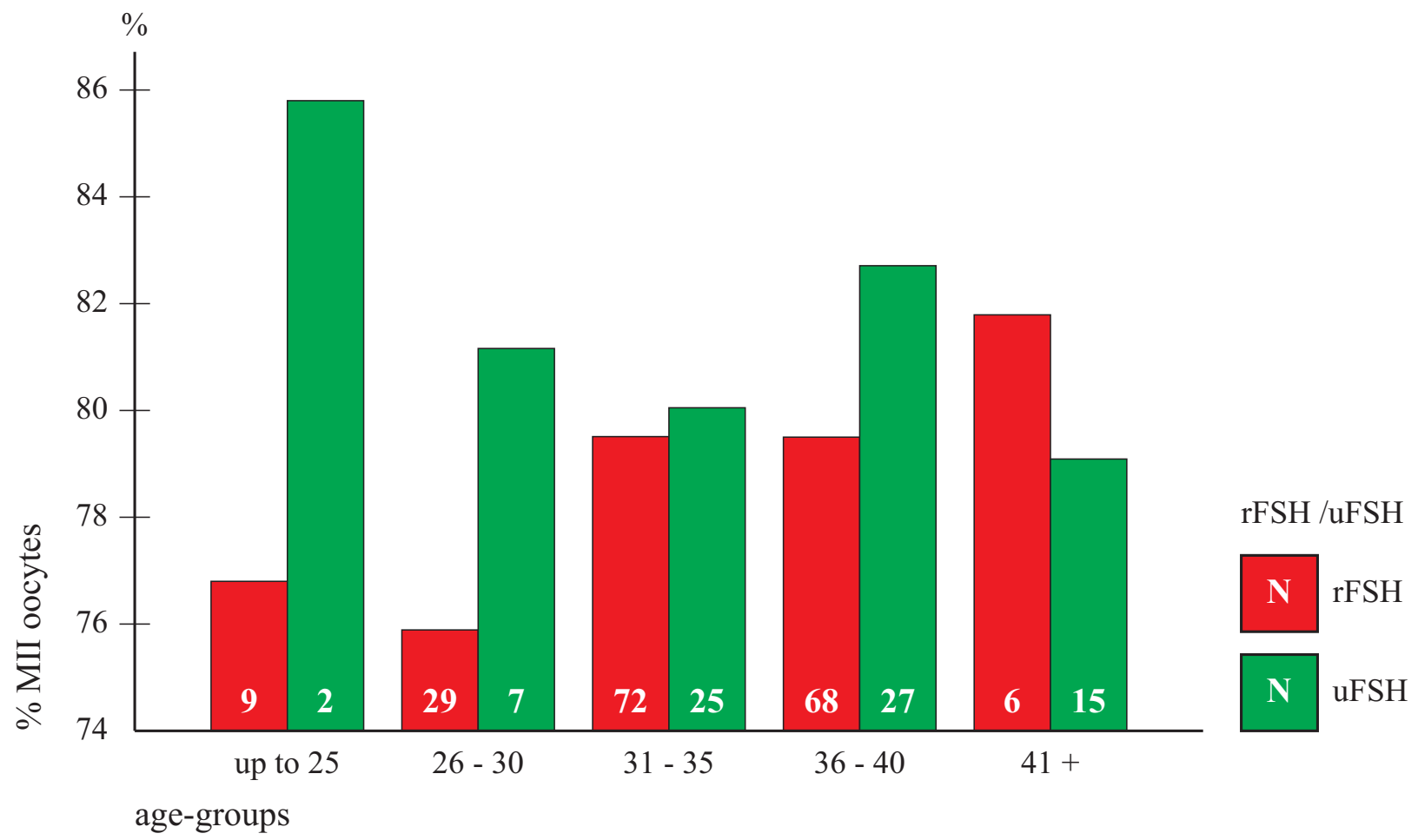

Fig. (4c). Comparison of mature (MII) oocytes retrieved after rFSH or uFSH stimulation. The percentage of mature oocytes was higher in the $\mathrm{uFSH}$ group than in the rFSH group, except in age group 41+, where the percentage in the uFSH group was lower than in the rFSH group. 
Fig. (4). Contd....

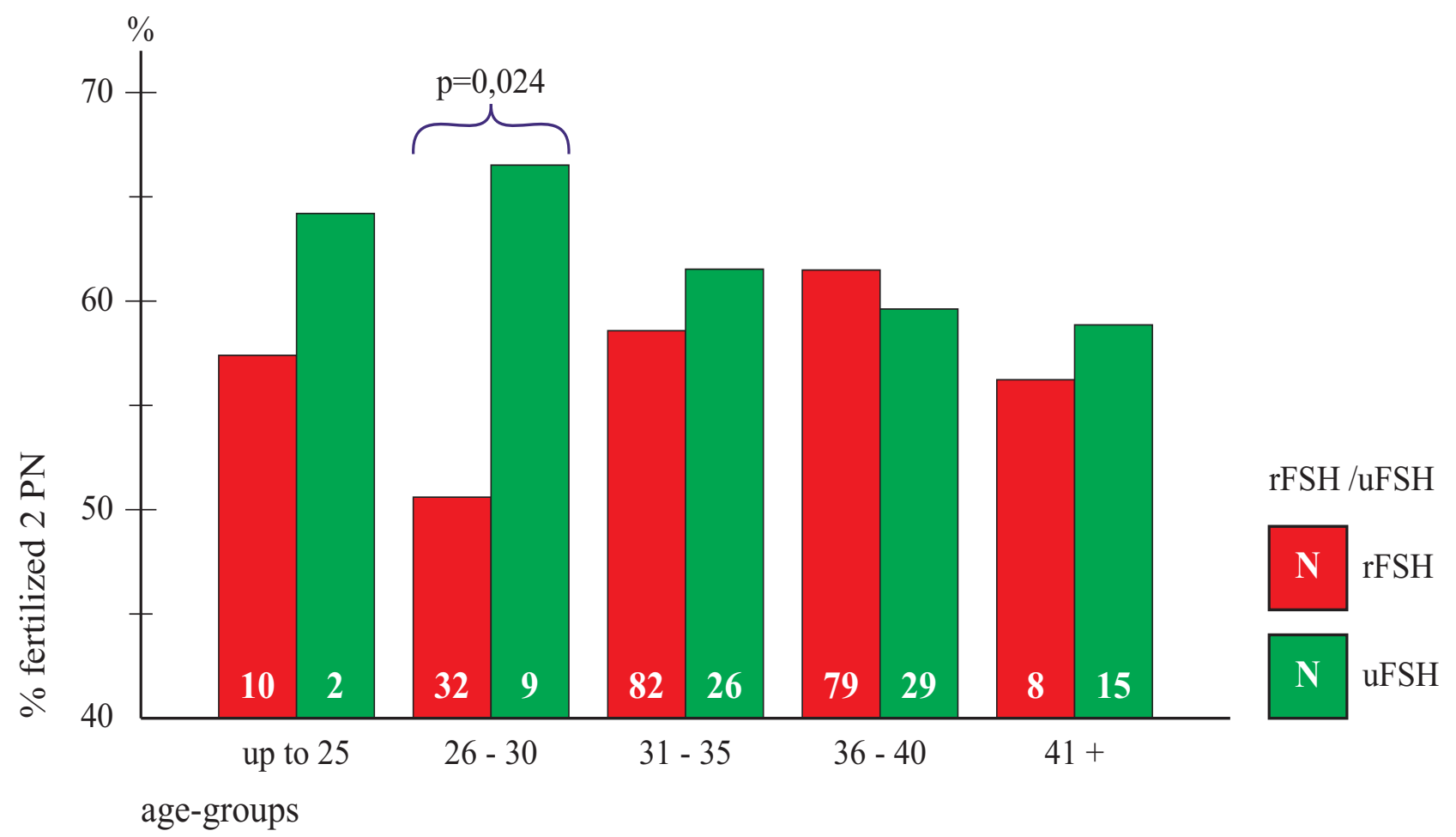

Fig. (4d). Comparison of fertilization rates after rFSH or uFSH stimulation. Higher fertilization rates than in the rFSH group were found in the uFSH group, except in age group 36-40 years, were the fertilization rate in the uFSH group was lower than in the rFSH group. In group 26 - 30years, the fertilization rate was significantly higher in the uFSH group compared to the rFSH- group $(\mathrm{p}=0,024)$.

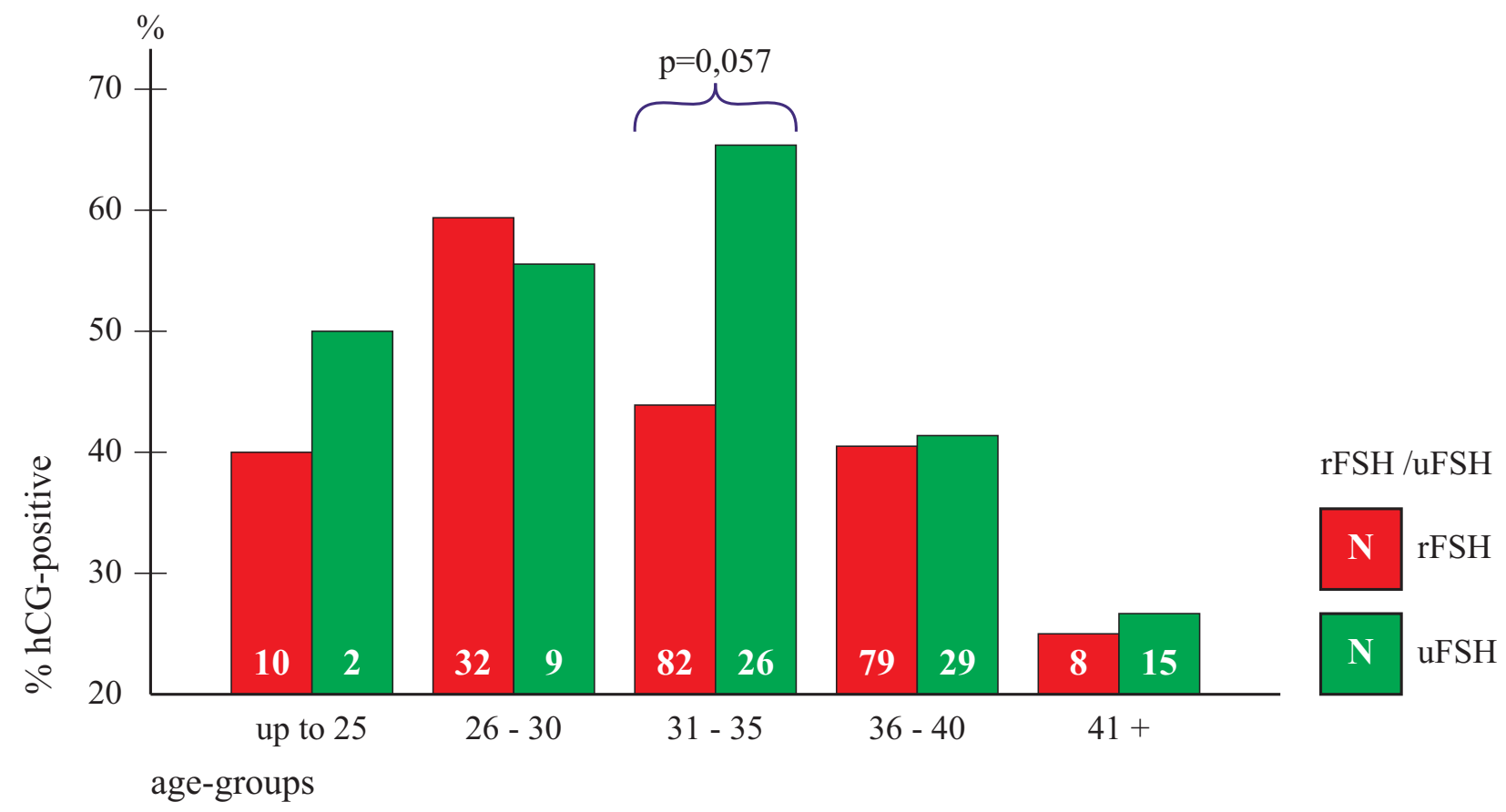

Fig. (4e). Comparison of hCG-positive pregnancies after rFSH or uFSH stimulation. Higher pregnancy rates than in the rFSH group were found in the uFSH group, except in age group 26-30 years, where the pregnancy rate in the uFSH group was lower than in the rFSH group. In age group $31-35$ years, the pregnancy rate in the uFSH group was just not significantly higher than in the rFSH group ( $\mathrm{p}=0,057)$. 
Fig. (4). Contd....

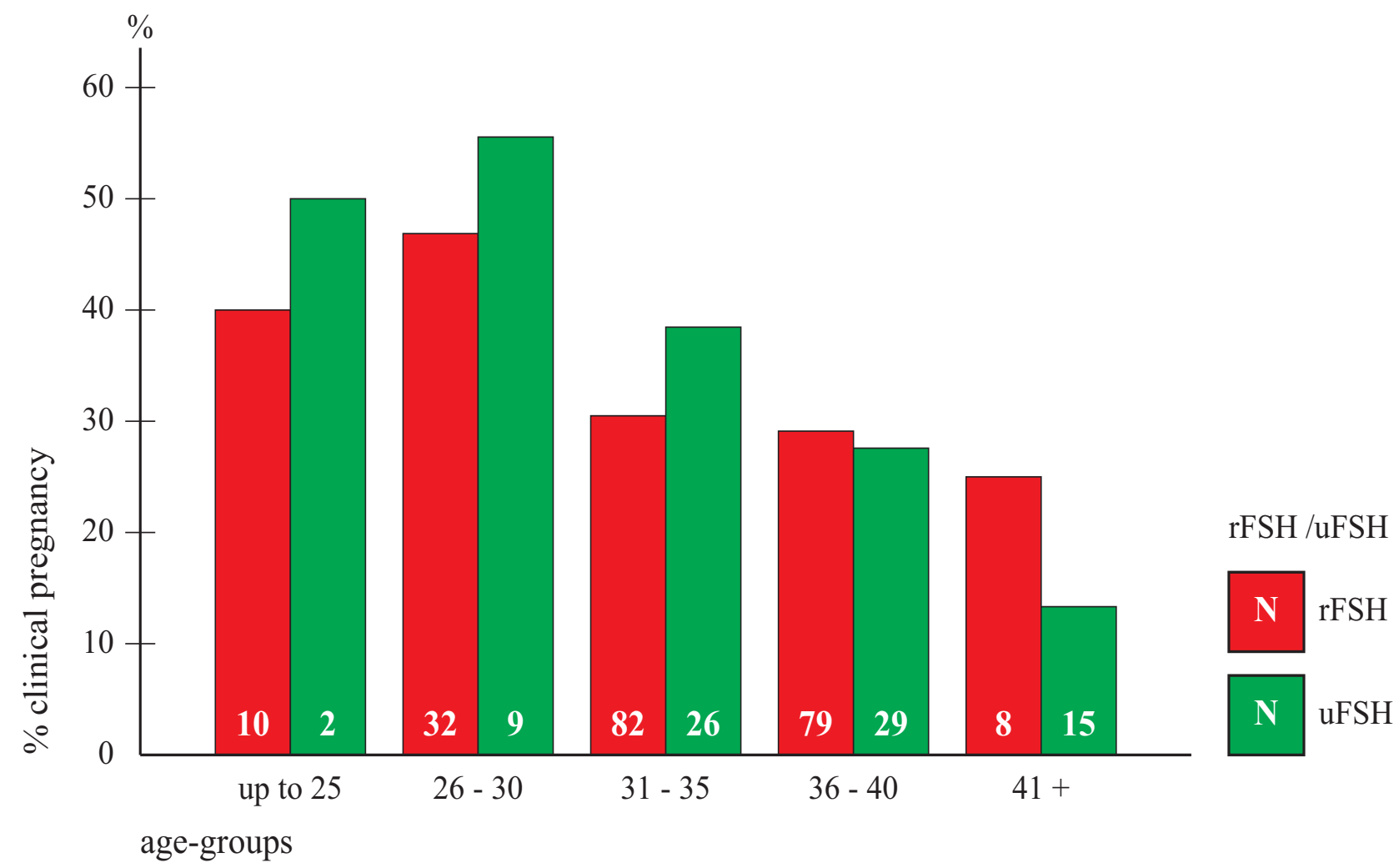

Fig. (4f). Comparison of clinical pregnancies after rFSH or uFSH stimulation. Rates were insignificantly higher in the uFSH group in younger patients up to 35 years and were insignificantly lower in the uFSH group in older patients over 35 years.

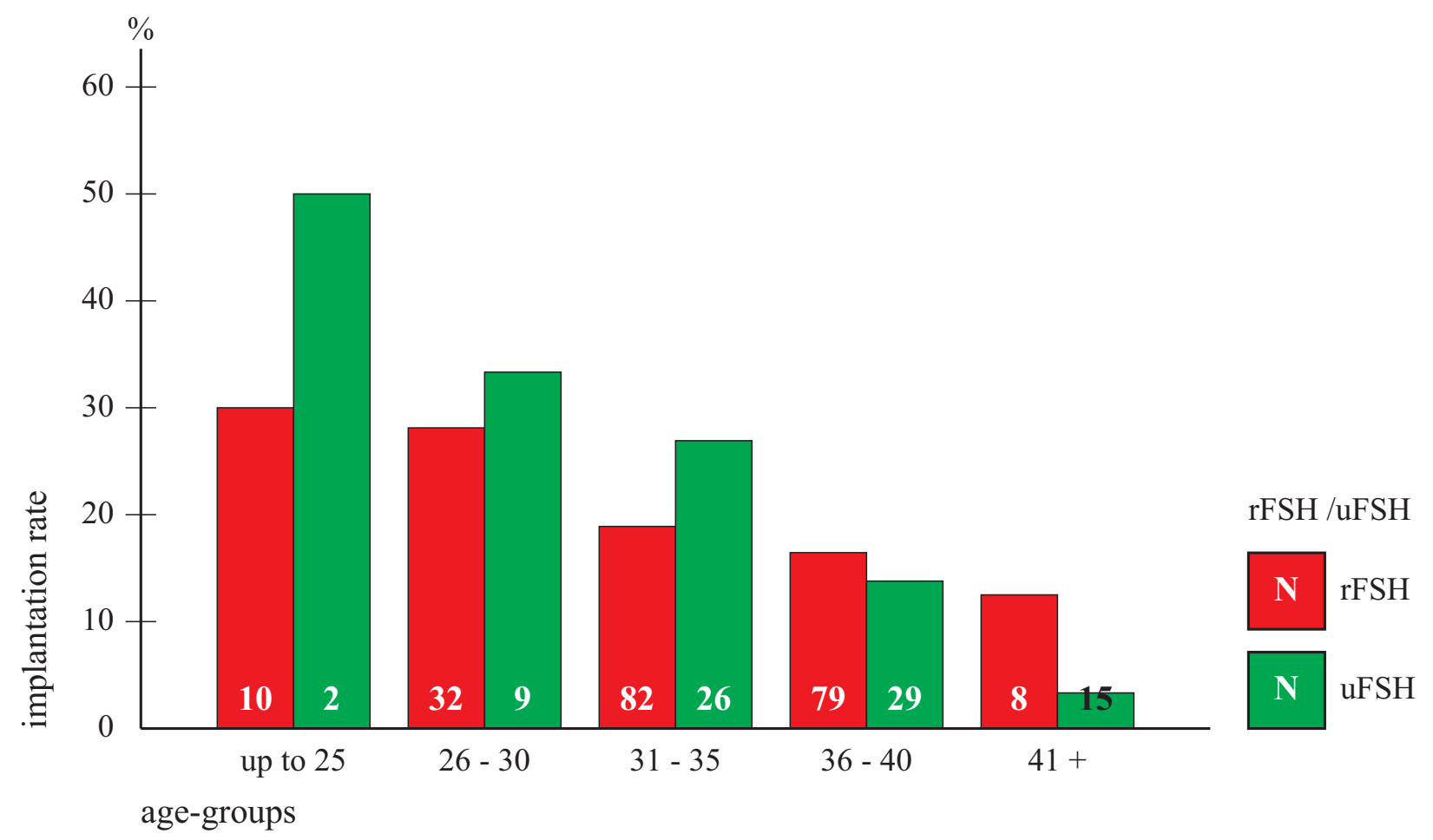

Fig. (4g). Comparison of implantation rates after rFSH or uFSH stimulation. Quite similar to figure 4f, implantation rates in the uFSH group were insignificantly higher in younger patients up to 35 years and were insignificantly lower in the uFSH group in older patients over 35 years. 
Table 4. Infertility-Relevant Basic Data and Gonadotropin Consumption in rFSH- or uFSH-Stimulated First-Ever IVF Cycles with > 10 Oocytes Retrieved and 2 Embryos Transferred

\begin{tabular}{|c|c|c|c|}
\hline Cause of Infertility & $\begin{array}{c}\text { rFSH Group } \\
\begin{array}{c}(\mathrm{n}=98) \\
\%\end{array}\end{array}$ & $\begin{array}{c}\text { uFSH Group } \\
(\mathbf{n}=\mathbf{2 7}) \\
\%\end{array}$ & $\mathbf{P}$ \\
\hline Male & 41.1 & 48.1 & \\
\hline Tubal & 13.7 & 18.5 & \\
\hline Functional & 32.6 & 25.9 & \\
\hline Mixed & 4.2 & & \\
\hline Idiopathic & 8.4 & 7.4 & NS \\
\hline Mean $\mathrm{BMI} \pm \mathrm{SD}$ & $23.2 \pm 4.4$ & $22.1 \pm 2.6$ & NS \\
\hline Mean duration of sterility $(y) \pm S D$ & $3.9 \pm 3.2$ & $2.9 \pm 1.9$ & NS \\
\hline $\begin{array}{l}\text { Cigarette smoking } \\
\text { No / day } \\
\text { None } \\
\text { up to } 10 \\
10-20 \\
>20\end{array}$ & $\begin{array}{l}76.7 \\
13.3 \\
10.0 \\
0.0\end{array}$ & $\begin{array}{l}84.0 \\
12.0 \\
4.0 \\
0.0\end{array}$ & NS \\
\hline $\begin{array}{l}\text { FSH / hMG consumption } \\
\text { Total IU FSH/cycle }(\mathrm{X} \pm \mathrm{SD}) \\
\text { Total IU hMG/cycle }(\mathrm{X} \pm \mathrm{SD})\end{array}$ & $\begin{array}{l}1634 \pm 304 \\
280 \pm 108\end{array}$ & $\begin{array}{l}1706 \pm 333 \\
319 \pm 117\end{array}$ & $\begin{array}{l}\text { NS } \\
\text { NS }\end{array}$ \\
\hline
\end{tabular}

\section{DISCUSSION}

We are well aware of the fact that a retrospective study is always distorted by confounding factors.

The first eye catching confounding point in our study regards the different antagonists: cetrorelix in Groups 1 and 3 and ganirelix in Group 2 (Figs. 1-3). Could this have deviated the final results?

Arguments against this assumption are, that in a prospective, randomized multicenter trial (17 centers) both, cetrorelix acetate and ganirelix acetate, suppressed LH to levels $<5 \mathrm{mU} / \mathrm{ml}$ in over $95 \%$ of cases and prevented $\mathrm{LH}$ surges $(>10 \mathrm{IU} / \mathrm{L})$ in all cases. In addition, similar safety profiles and IVF and ET outcomes were assessed in both groups [42]. Another argument against the concern is the fact that in our study the consumption of FSH and HMG ampoules was nearly the same in the rFSH and uFSH group (Table 4), indicating, that - according to follicular growth there was no need to stimulate one group more than the other with gonadotropins. It is therefore very unlikely that the different $\mathrm{GnRH}$-antagonists in the $\mathrm{rFSH}$ protocols would have caused any relevant deviation of the results.

The same holds true for the skepticism about the additional administration of HMG from day 6: Could this have confounded the study. We do not think so, because every patient in both groups received exactly 75 IU of HMG daily from day 6 onwards up to the day of hCG administration.

Accordingly, the HMG consumption between the groups was balanced (Table 4).

Could the aggregation of Group I (Follitropin alfa) with Group II (Follitropin beta) have confounded the study? We do not think so, because no differences in effect between the preparations have been found so far $[43,44]$ and we could not find differences between Groups I and II either (Table 2).

In contrast to the mentioned factors, however, there are indeed powerful confounding parameters, which have to be taken into consideration. We could demonstrate by logistic regression analysis that the following parameters have a strong influence on the pregnancy rate: age of the patient, number of embryos transferred, total number of IVF-cycles performed so far, and the number of oocytes retrieved (Table 1). Taking this into account, we included for the analysis only first-ever IVF cycles where 2 embryos had been transferred and stratified age into rather narrow strata. However, the study groups were still not comparable, because there were significantly more oocytes in the $\mathrm{rFSH}$ group than in the uFSH group, and the difference was more pronounced in the older patients (Fig. 4b). Comparable groups were finally created by performing a further selection: only patients who had more than 10 oocytes retrieved were included (Table $\mathbf{5}$ ). Although the groups were now relatively low in number, they were comparable with respect to age, number of eggs retrieved, number of embryos replaced and in that they had their first IVF treatment in life.

Although no significant differences were found between the $\mathrm{rFSH}$ and $\mathrm{uFSH}$ group, it is worth noting, that the following reproductive parameters were in favor of the uFSH group: rates of oocyte maturation, fertilization, embryo score 4 , embryo cryopreservation, pregnancy, clinical pregnancy, implantation and abortion (Table 5). That means, that all end points go in the same direction and the cryopreservation rate and abortion rate support additionally our conclusions. 
Table 5. Results of Antagonist Protocols for First-Ever IVF Cycles with $>10$ Oocytes Retrieved and 2 Embryos Transferred, Stimulated with Either rFSH or uFSH

\begin{tabular}{|c|c|c|c|}
\hline & rFSH Group & uFSH Group & $\mathbf{P}$ \\
\hline Oocytes $(\mathrm{X} \pm \mathrm{SD})$ & $15.6 \pm 3.9$ & $14.4 \pm 2.9$ &, 171 \\
\hline Mature (MII) Oocytes (\%) & $76.5 \pm 16.3$ & $79.0 \pm 13.6$ & ,485 \\
\hline Fertil. 2 PN ICSI (\%) & $68.9 \pm 16.1$ & $72.4 \pm 21.9$ &, 394 \\
\hline Embryo Score $4(\%)$ & $25.7 \pm 22.4$ & $31.1 \pm 28.3$ & ,299 \\
\hline Endometrial thickness $(\mathrm{X} \pm \mathrm{SD})$ & $11.0 \pm 2.3$ & $11.0 \pm 1.3$ & 1,00 \\
\hline Pregnancy rate $(\%)$ & $43.9 \pm 49.9$ & $59.3 \pm 50.1$ &, 159 \\
\hline Abortion rate (\%) & $14.0 \pm 35.1$ & $12.5 \pm 34.2$ &, 887 \\
\hline Cryopreserved embryos (\%) & $19.3 \pm 24.7$ & $30.5 \pm 42.8$ & ,085 \\
\hline
\end{tabular}

These findings are consistent with the results of a study where highly purified menotropin (hphMG) was compared with $\mathrm{rFSH}$ in antagonist cycles with compulsory singleblastocyst transfer [25]. In this randomized, assessor-blind, parallel group, multicenter ( 25 centers in 7 countries) noninferiority trial, also significantly fewer oocytes were retrieved in the hphMG group, yet, the results of hCG-positive pregnancy, clinical pregnancy, ongoing pregnancy and live birth were in favor of hphMG, but the differences were not significant. The authors came to the conclusion, that hphMG is at least as effective as rFSH in antagonist cycles, and the robustness of this conclusion was supported by the consistency of findings across all of the populations analyzed.

In addition, there is ample evidence from basic research that $\mathrm{rFSH}$ and $\mathrm{uFSH}$ have different modes of action. uFSH belongs to the urinary based FSH preparations, which are more glycosylated and more acidic than the less glycosylated and less acidic recombinant FSH preparations, Follitropin alfa and beta. Isoelectric focusing of batches of Urofollitropin and Follitropin alfa demonstrated marked differences between the preparations, with pI 3.0 - 5.2 (acidic) for Urofollitropin and $\mathrm{pI} 4.0$ - 5.85 (less acidic) for Follitropin alfa [45]. Nayudu, et al. (2002) [46] cultured isolated intact mouse follicles, a method which allows observation of the sequential events of follicle development up to the antral stage, and stimulated these follicles with various doses of FSH isoforms. They found differences in the threshold and tolerance doses of the isoforms: 0.5 and $2.5 \mathrm{ng} / \mathrm{ml}$ respectively for the less acidic, 5.0 and $10 \mathrm{ng} / \mathrm{ml}$ respectively for the mid-acidic and 10 and $50 \mathrm{ng} / \mathrm{ml}$ respectively for the acidic isoform. In other words, the follicles were more sensitive to the less acidic isoforms but tolerated them only in a quite narrow range. In contrast, while follicles were less sensitive to the acidic isoforms, they tolerated them in a wider range of doses. In addition, follicles stimulated with acidic
FSH required 5 days to reach the dimensions recorded at day 3 with the least acidic FSH [47].

It would therefore appear that the acidic isoforms stimulate the ovarian follicles in a more physiological manner and are more selective for good quality oocytes, especially in the early phase of follicular maturation. Our results may provide some evidence to support this theory, because the final results showed that the uFSH group had a trend towards better outcome results compared to the rFSH, in first-ever IVF cycles of patients having $>10$ oocytes and two embryos transferred (Table 5).

\section{CONCLUSION}

In a retrospective study with antagonist cycles, $\mathrm{rFSH}$ preparations (Follitropin alfa and Follitopin beta) were compared to a new uFSH preparation (Urofollitropin) with the following end points: hCG-positive pregnancy rate, number of oocytes retrieved, number of mature oocytes (MII), fertilization rate, embryo quality score 4, endometrial thickness, clinical pregnancy rate, and implantation rate.

The results showed that stimulation with uFSH resulted in a lower yield of oocytes compared to $\mathrm{rFSH}$. Nevertheless, uFSH proved to be at least as effective as rFSH in younger patients in regard to the end points of the study, except the number of oocytes. The higher cryopreservation rate and the lower abortion rate in the $\mathrm{uFSH}$-group also supported the concept, that uFSH produces fewer oocytes but oocytes with a better quality compared to $\mathrm{rFSH}$. According to ample basic research, this mode of action is attributed to the fact that $\mathrm{uFSH}$ is more glycosylated and more acidic than $\mathrm{rFSH}$.

However, the clinical results should be confirmed by well designed prospective randomized studies. 


\section{CONFLICT OF INTEREST}

The authors confirm that this article content has no conflicts of interest.

\section{ACKNOWLEDGEMENTS}

The authors thank Prof. Dr. Anselm Eder, Department of Statistics, Institute of Sociology, University of Vienna, Austria, for supervising the statistical analysis. The authors also cordially thank Dr. Sharon Mortimer, Oozoa Biomedical Inc, Canada, for her critical contributions and her linguistic support in preparing the manuscript.

\section{REFERENCES}

[1] Lunenfeld B. Development of gonadotropins for clinical use. Reprod BioMed Online 2001; 4(suppl. 1): 11-7.

[2] Rathnam P, Saxena BB. Primary amino acid sequence of follicle stimulating hormone from human pituitary glands. J Biol Chem 1975; 17: 6735-42.

[3] Chappel SC, Ulloa-Aguirre A, Ramaley J. Sexual maturation in female rats: time- related changes in the isoelectric focusing pattern of anterior pituitary follicle-stimulating hormone. Biol Reprod 1983; 28: 196-205.

[4] Padmanabhan V, Lang LL, Sonstein J, et al. Modulation of serum follicle-stimulating hormone bioactivity and isoform distribution by estrogenic steroids in normal women and in gonadal dysgenesis. $\mathrm{J}$ Clin Endocr Metab 1988; 67: 465-73.

[5] Wide L. Follicle-stimulating hormones in anterior pituitary gland from children and adults differ in relation to sex and age. J Endocrinol 1989; 123: 519-29.

[6] Padmanabhan V, Sairam MR, Hassing JM, et al. Follicle stimulating hormone signal transduction: role of carbohydrate in aromatase induction in immature Sertoli cells. Mol Cell Endocrinol 1991; 79: 119-28.

[7] D'Antonio M, Borrelli F, Datola A, et al. Biological characterization of recombinant human follicle stimulating hormone isoforms. Hum Reprod 1999; 14: 1160-7.

[8] Out HJ, Mannaerts BM, Driessen SG, et al. A prospective, randomized, assessor-blind, multicentre study comparing recombinant and urinary follicle stimulating hormone (Puregon vs. Metrodin) in invitro fertilization. Hum Reprod 1995; 10: 2534-40.

[9] Out HJ, Driessen SG, Mannaerts BM, et al. Recombinant folliclestimulating hormone (follitropin beta, Puregon) yields higher pregnancy rates in in vitro fertilization than urinary gonadotropins. Fertil Steril 1997; 68: 138-42.

[10] Raga F, Bonilla-Musoles F, Casañ EM, et al. Recombinant follicle stimulating hormone stimulation in poor responders with normal basal concentrations of follicle stimulating hormone and oestradiol: improved reproductive outcome. Hum Reprod 1999; 14: 1431-4.

[11] De Placido G, Alviggi C, Mollo A et al. Recombinant follicle stimulating hormone is effective in poor responders to highly purified follicle stimulating hormone. Hum Reprod 2000; 15: 17-20.

[12] Lenton E, Soltan A, Hewitt J, et al. Induction of ovulation in women undergoing assisted reproductive techniques: recombinant human FSH (follitopin alpha) vs. highly purified urinary FSH (urofollitopin HP). Hum Reprod 2000; 15: 1021-27.

[13] Daya S. Updated meta-analysis of recombinant follicle-stimulating hormone (FSH) vs. urinary FSH for ovarian stimulation in assisted reproduction. Fertil Steril 2002; 77: 711-4.

[14] Strehler E, Abt M, El-Danasouri I, et al. Impact of recombinant follicle-stimulating hormone and human menopausal gonadotrophins on in vitro fertilization outcome. Fertil Steril 2001; 75: 332-6.

[15] Ng EHY, Lau EYL, Yeung WSBY, et al. HMG is as good as recombinant human FSH in terms of oocyte and embryo quality: a prospective randomized trial. Hum Reprod 2001; 2: 319-25.

[16] Al-Inany HG, Aboulghar M, Mansour R, et al. Meta-analysis of recombinant vs. urinary- derived FSH: an update. Hum Reprod 2003; 18: 305-13.

[17] van Wely M, Bayram N, van der Veen F. Recombinant FSH in alternative doses or $v s$. urinary gonadotrophins for ovulation induction in subfertility associated with polycystic ovary syndrome: a systematic review based on a Cochrane review. Hum Reprod 2003; 18: 1143-9.
[18] van Wely m, Kwan I, Burt AL, et al. Recombinant vs.. urinary gonadotrophin for ovarian stimulation in assisted reproductive technology cycles (Review). Cochrane Database Syst Rev; 2011; 16(2): CD005354.

[19] Revelli A, Poso F, Gennarelli G, et al. Recombinant vs. highlypurified, urinary follicle-stimulating hormone (r-FSH vs. HP$\mathrm{uFSH}$ ) in ovulation induction: a prospective, randomized study with cost-minimization analysis. Reprod Biol Endocr 2006; 4: 1-7.

[20] Jee BC, Suh CS, Kim YB, et al. Kim SH, Moon SY. Clinical efficacy of highly purified hMG vs. recombinant FSH in IVF/ICSI cycles: a meta-analysis. Gynecol Obstet Invest 2010; 70: 132-7.

[21] Westergaard LW, Bossuyt PM, Van der Veen F, et al. Human menopausal gonadotropin $v s$. recombinant follicle stimulation hormone for ovarian stimulation in assisted reproductive cycles. Cochrane Database Syst Rev 2011; 16: CD003973.

[22] Ziebe S, Lundin K, Janssens R, et al. Influence of ovarian stimulation with HP-hMG or recombinant FSH on embryo quality parameters in patients undergoing IVF. Hum Reprod 2007; 22: 2404-13.

[23] Coomarasamy A, Afnan M, Cheema D, et al. Urinary hMG vs. recombinant FSH for controlled ovarian hyperstimulation following an agonist long down-regulation protocol in IVF or ICSI treatment: a systemic review and meta-analysis. Hum Reprod 2008; 23: 3105.

[24] Pacchiarotti A, Sbracia M, Frega A, et al. Urinary hMG (Menopur) vs. recombinant FSH plus recombinant LH (Pergoveris) in IVF: a multicenter, prospective, randomized controlled trial. Fertil Steril 2010; 94: 2467-9.

[25] Devroey P, Pellicer A, Andersen AN, et al. A randomized assessorblind trial comparing highly purified $\mathrm{hMG}$ and recombinant FSH in a GnRH antagonist cycle with compulsory single-blastocyst transfer. Fertil Steril 2012; 97: 561-71.

[26] Selman HA, De Santo M, Sterzik K, et al. Effect of highly purified urinary follicle stimulating hormone on oocyte and embryo quality. Fertil Steril 2002; 78: 1061-7.

[27] Mohamed MA, Sbracia M, Pacchiarotti A, et al. Urinary folliclestimulating hormone (FSH) is more effective than recombinant FSH in older women in a controlled randomized study. Fertil Steril 2006; 85: 1398-403.

[28] Pacchiarotti A, Aragona C, Gaglione R, et al. Efficacy of a combined protocol of urinary and recombinant follicle-stimulating hormone used for ovarian stimulation of patients undergoing ICSI cycle. J Assist Reprod Genet 2007; 24: 400-5.

[29] Abate A, Nazzaro A, Salerno A, et al. Efficacy of recombinant vs. human derived follicle stimulating hormone on the oocyte and embryo quality in IVF-ICSI cycles: randomised, controlled, multicentre trial. Gynecol Endocrinol 2009; 25: 479-84.

[30] Aboulghar M, Saber W, Amin Y, et al. Prospective, randomized study comparing highly purified urinary follicle-stimulating hormone (FSH) and recombinant FSH for in vitro fertilization/intracytoplasmic sperm injection in patients with polycystic ovary syndrome. Fertil Steril 2010; 94: 2332-4.

[31] Kemeter P, Feichtinger W. Prednisolone supplementation to clomid and/or gonadotrophin stimulation for in-vitro fertilization - a prospective randomized trial. Hum Reprod 1986; 1: 441-4.

[32] Kemeter P. The usefulness of corticoids in stimulated cycles for in vitro fertilization - Editorial. J In Vitro Fertl Embryo Transf 1987; 4: 69-72.

[33] Marrs R, Meldrum D, Muasher S, et al. Randomized trial to compare the effect of recombinant human FSH (follitropin alfa) with or without recombinant human $\mathrm{LH}$ in women undergoing assisted reproduction treatment. Reprod Biomed Online 2003; 8: 175-82.

[34] Humaidan P, Bungum M, Bungum L, et al. Effects of recombinant LH supplementation in women undergoing assisted reproduction with GnRH agonist down-regulation and stimulation with recombinant FSH: an opening study. Reprod Biomed Online 2004; 8: 63543.

[35] Bosch E, Vidal C, Labarta E, et al. Highly purified hMG vs. recombinant FSH in ovarian hyperstimulation with GnRH antagonists - a randomized study. Hum Reprod 2008; 23: 2346-51.

[36] Matorras R, Prieto B, Exposito A, et al. Mid-follicular LH supplementation in women aged 35-39 years undergoing ICSI cycles: a randomized controlled study. Reprod Biomed Online 2009; 19: 879-87.

[37] Ferraretti AP, Gianaroli L, Magli MC, et al. Exogenous luteinizing hormone in controlled ovarian hyperstimulation for assisted reproduction techniques. Fertil Steril 2004 82:1521-6. 
[38] De Placido G, Alviggi C, Perino A, et al. Recombinant human LH supplementation $v s$. recombinant human FSH (rFSH) step-up protocol during controlled ovarian stimulation in normogonadotrophic women with initial inadequate ovarian response to rFSH. A multicentre, prospective, randomized controlled trial. Hum Reprod 2005; 2: 390-6.

[39] Ruvolo G, Bosco L, Pane A, et al. Lower apoptosis rate in human cumulus cells after administration of recombinant luteinizing hormone to women undergoing ovarian stimulation for in vitro fertilization procedures. Fertil Steril 2007; 87: 542-5.

[40] Barrenetxea G, Agirregoikoa JA, Jimenez MR, et al. Ovarian response and pregnancy outcome in poor responder women: a randomized controlled trial on the effect of luteinizing hormone supplementation on in vitro fertilization cycles. Fertil Steril 2008; 89: 546-53.

[41] Bongso A. Handbook on blastocyst culture. Singapore: Sydney Press Indusprint (s) Pte Ltd, 1999.

[42] Wilcox J, Potter D, Moore M, et a. Prospective randomized trial comparing cetrorelix acetate and ganirelix acetate in a programmed, flexible protocol for premature luteinizing hormone surge prevention in assisted reproductive technologies. Fertil Steril 2005; 84: 108-17.

[43] Fried G, Harlin J, Wramsby H. Recombinant FSH - clinical experience with two different preparations. Fertil Steril 1998; 70(suppl. 1): 112.

[44] Brinsden P, Akagbosu F, Gibbons L, et al. A comparison of the efficacy and tolerability of two recombinant human folliclestimulating hormone preparations in patients undergoing in vitro fertilization-embryo transfer. Fertil Steril 2000; 73: 114-6.

[45] Lispi M, Bassett R, Crisci C, et al. Comparative assessment of the consistency and quality of a highly purified FSH extracted from urine (urofollitropin) and a recombinant human FSH (follitropin a). Reprod Biomed Online 2006; 13: 179-93.

[46] Nayudu PL, Vitt UA, Barrios de Tomasi J, et al. Intact follicle culture: what it can tell us about the role of FSH glycoforms during follicle development. Reprod Biomed Online 2002; 5: 240-53.

[47] Vitt UA, Kloosterboer HJ, Rose UM, et al. Isoforms of human recombinant follicle-stimulating hormone: comparison of effects on murine follicle development in vitro. Biol Reprod 1998; 59: 85461

Received: June 19, 2013

Revised: July 19, 2013

Accepted: July 25, 2013

(C) Kemeter et al.; Licensee Bentham Open.

This is an open access article licensed under the terms of the Creative Commons Attribution Non-Commercial License (http:/creativecommons.org/licenses/by-nc/3.0/) which permits unrestricted, non-commercial use, distribution and reproduction in any medium, provided the work is properly cited. 